\title{
El estudio de la estructura atómica con modelos tridimensionales y la web 2.0 mejoran el desempeño de estudiantes de educación media en pruebas específicas
}

\section{The use of both three-dimensional models of atomic atructure and Web 2.0 improve the performance of high school students in specific tests}

\section{Die verwendung sowohl dreidimensionale modelle der atomaren struktur und Web 2.0 verbessern die leistung der gymnasiasten in spezifische tests}

\author{
Benjamín Dussán Díaz ${ }^{1}$, Andrés Felipe Flórez Rivera², Manuel García Flórez ${ }^{3}$
}

\section{Resumen}

Para mejorar la comprensión del concepto de átomo en estudiantes de educación media se han comparado dos estrategias didácticas diferentes de la metodología tradicional. Este concepto es ampliamente usado en disciplinas como química, biología, biofísica, bioquímica, entre otras, y es una base sobre la cual se construyen diferentes procesos de racionamiento. Fueron organizados tres grupos experimentales: 1) Grupo de Docencia Tradicional al que se le transmitió el tema de forma oral (con ayuda de un libro guía); 2) Grupo Modelos Físicos Tridimensionales, en el que se realizaron las clases con modelos didácticos del átomo, y; 3) Grupo Tecnología de la Información, grupo donde se usaron las herramientas de la web 2.0. El desempeño de cada estudiante se valoró a través de una prueba escrita compuesta de 12 preguntas relacionadas con el tema, elaboradas y clasificadas con base en la taxonomía cognitiva de Bloom. Los análisis de correlación indican un mejor desempeño en las evaluaciones de los estudiantes pertenecientes al Grupo Modelos Físicos Tridimensionales, seguidos del Grupo Tecnología de la Información, mientras que el grupo de Docencia Tradicional obtuvo las calificaciones más bajas. Los resultados obtenidos sugieren que es importante explorar nuevas alternativas de docencia, y que específicamente al trasmitir el concepto del átomo, el uso de Modelos Tridimensionales se presenta como una estrategia útil que puede contribuir con una mejor comprensión por parte de los estudiantes.

\begin{abstract}
To improve the comprehension of the Atom Concept in High School students, two different didactical strategies have been compared that belong to the traditional methodology. This concept is broadly used in study disciplines such as: Chemistry, Biology, Biophysics, Biochemistry, amongst others. It is a foundation on which it is possible to build different process of thinking.
\end{abstract}

Three experimental groups were organized: 1) Traditional Teaching Group to which the main subject was transmitted by oral way (with the aid of a guidebook); 2) Physic Tridimensional Models Group, in which the classes were taught with didactical models of the Atom, and 3) Information Technologies Group, group in which web tools 2.0 were used.

The performance of each student was evaluated through a written test that was made from 12 questions, related to the main subject and classified on the basis of the Bloom's Cognitive Taxonomy.

The correlation analysis indicated a better performance in the evaluations of students that belonged to the Physic Tridimensional Models Group, followed by the Information Technologies Group. The traditional teaching group obtained the lowest grades.

Keywords: comprehension, atom, concept, didactical strategies, students.

Palabras claves: comprensión, concepto, átomo, didáctica, estudiantes.

1 - Magíster en Educación. Universidad Surcolombiana. Neiva. Colombia. benjamindus@hotmail.com

2 - Programa de Maestría en Estadística. Universidad Nacional de Colombia. Medellín. Colombia.

3 - Doctor en Ciencias Biológicas. Universidad Surcolombiana. Neiva.Colombia. garcia@usco.edu.co 


\section{Zusammenfassung}

Um das Verständnis der Atom-Konzept in der High School Schüler zu verbessern, wurden zwei verschiedene didaktische Strategien verglichen worden, die zu den traditionellen Methoden gehören. Dieses Konzept wird im Großen und Ganzen in der Studie Disziplinen wie: Chemie, Biologie, Biophysik, Biochemie, unter anderem. Es ist eine Grundlage, auf der es möglich ist, unterschiedliche Denkprozess aufzubauen.

Drei Versuchsgruppen wurden organisiert: 1) traditionelle Lehrgruppe, denen das Hauptmotiv wurde von oralen Weg mit Hilfe eines Leitfadens übertragen (); 2) Physic Tridimensional Models Group, in der die Klassen wurden mit didaktischen Modelle der Atom gelehrt, und 3) Information Technologies Group, Gruppe, in der Web-Tools 2.0 verwendet wurden.

\section{Introducción}

El aumento del conocimiento sobre la estructura molecular de componentes de la célula, ha estimulado el desarrollo de nuevas técnicas de enseñanza donde se emplean representaciones en tres dimensiones que buscan mejorar la comprensión del estudiante (Kramer, Dahmani, Delouche, Bidabe, \& Schneeberger, 2012).

Sin embargo, en la docencia tradicional predomina el ambiente pasivo y con exposiciones magistrales como principal herramienta, se trasmiten procesos complejos, dinámicos y frecuentemente microscópicos (Modell, 1996; O'Dowd \& Aguilar-Roca, 2009).

Bajo esta metodología, estudiantes y docentes consideran exitoso el proceso de aprendizaje ya que el alumno sigue la lógica de la clase y memoriza eventos plasmados en un libro guía, pero el desempeño en las pruebas, en su mayoría, muestra un conocimiento superficial de conceptos fundamentales de la materia (O'Dowd \& Aguilar-Roca, 2009).

Una de las mayores dificultades en docencia es la transmisión de conocimientos a través de la palabra escrita o hablada, sin el adecuado apoyo visual, lo que constituye un reto al momento de enseñar temas abstractos como los conceptos de química (Pérez Miranda, 1995).

En relación a estas dificultades, (Escalona \& Fontal, 2008), afirman que la enseñanza de química se realiza de forma deficiente; hay problemas de comprensión; no hay coherencia entre contenido del currículo y su evaluación. Además, se presentan altos niveles de deserción cuando es disciplina optativa, siendo notoria la disminución del número de jóvenes interesados en la química como carrera.

Contribuye con esto quizá, que es considerada como ciencia difícil que a pesar de ser concreta se fundamenta en conceptos abstractos, como el concepto de átomo el cual es intangible (Adúriz-Bravo \& IzquierdoAymerich, 2009; Mercè, 2004) (Cárdenas, 2006).

El estudiante debe tomar un papel activo y entender que las estructuras a nivel molecular tienen relación directa con la función. Por ejemplo, la estructura de las proteínas tiene relación directa con su procesamiento en las vías bioquímicas y celulares. La dificultad radica en la baja comprensión de estructuras que son invisibles por su dimensión (Harris et al., 2009).

En esta investigación se buscó una metodología económica y de fácil aplicación en estudiantes de educación media que permita mejorar su desempeño al momento de analizar, comprender, sintetizar, conocer y aplicar conceptos, de temas considerados como abstractos.

Para tal fin se usaron dos estrategias diferentes a la docencia tradicional, para trabajar el tema estructura atómica. Los resultados indican una mayor comprensión con el uso de técnicas diferentes a la docencia tradicional como los modelos tridimensionales, y la web 2.0.

\section{Metodología}

El estudio fue realizado en el segundo semestre del año 2010 en la Institución Educativa de Departamental Tierra de Promisión, institución de carácter público localizada en la zona urbana de la ciudad de Neiva, en el departamento del Huila, Colombia. La muestra de la investigación comprendió 71 estudiantes de grado decimo, entre los 14 y 18 años de edad.

Se asume que todos los estudiantes poseen el conocimiento previo para abordar los temas tratados ya que provienen del grado noveno y todos ellos aprobaron los requisitos para iniciar el grado décimo.

A pesar de usar diferentes metodologías, todos los estudiantes recibieron la unidad estructura atómica, en seis sesiones de 55 minutos de duración ( 5 teóricas y una de evaluación), en las que se presentaron los diferentes subtemas relacionados (tabla 1).

Tabla 1. Temáticas abordadas durante las sesiones

\begin{tabular}{ll}
\hline Sesión & TEMA \\
\hline 1 & $\begin{array}{l}\text { Modelo atómico de Leuclpo y Demócrito, Modelo } \\
\text { atómico de John Dalton }\end{array}$ \\
\hline 2 & Modelo atómico de Joseph J. Thompson \\
\hline 3 & Modelo atómico de Rutherford \\
\hline 4 & Modelo atómico Niels Bohr \\
\hline 5 & Modelo atómico actual: mecánico - ondulatorio \\
\hline 6 & Evaluación \\
\hline
\end{tabular}


Los estudiantes fueron distribuidos aleatoriamente, en los grupos descritos a seguir; 1) Grupo Docencia Tradicional (GT): formado por 37 estudiantes, recibieron clases magistrales, cada estudiante al capítulo Estructura Atómica del libro Química $10^{\circ}$ de Poveda, (págs. 75 a 93). El uso de este libro se justifica por tratarse del texto guía de la disciplina; 2) Grupo Modelos Físicos Tridimensionales (GFT): formado por 17 estudiantes, fueron usados modelos didácticos que representan modelos atómicos (Tabla 2). El tercer grupo, 3) Grupo Tecnología de la Información y la
Comunicación (GTIC), utilizaron las tecnologías de la información y la comunicación, el dominio http: //www.wikispaces.com, donde elaboraron una Wiki con los contenidos respectivos para cada sesión iguales a los desarrollados por los otros dos grupos. Cada estudiante, de este grupo, tuvo acceso libre a un computador equipado con Monitor de 17 pulgadas, mouse y teclado, cajas de sonido, con sistema operativo Windows XP, programa office 2007 y con acceso libre a internet de $2000 \mathrm{~K}$.

Tabla 2. Modelos usados durante las actividades del Grupo Modelos Físicos Tridimensionales

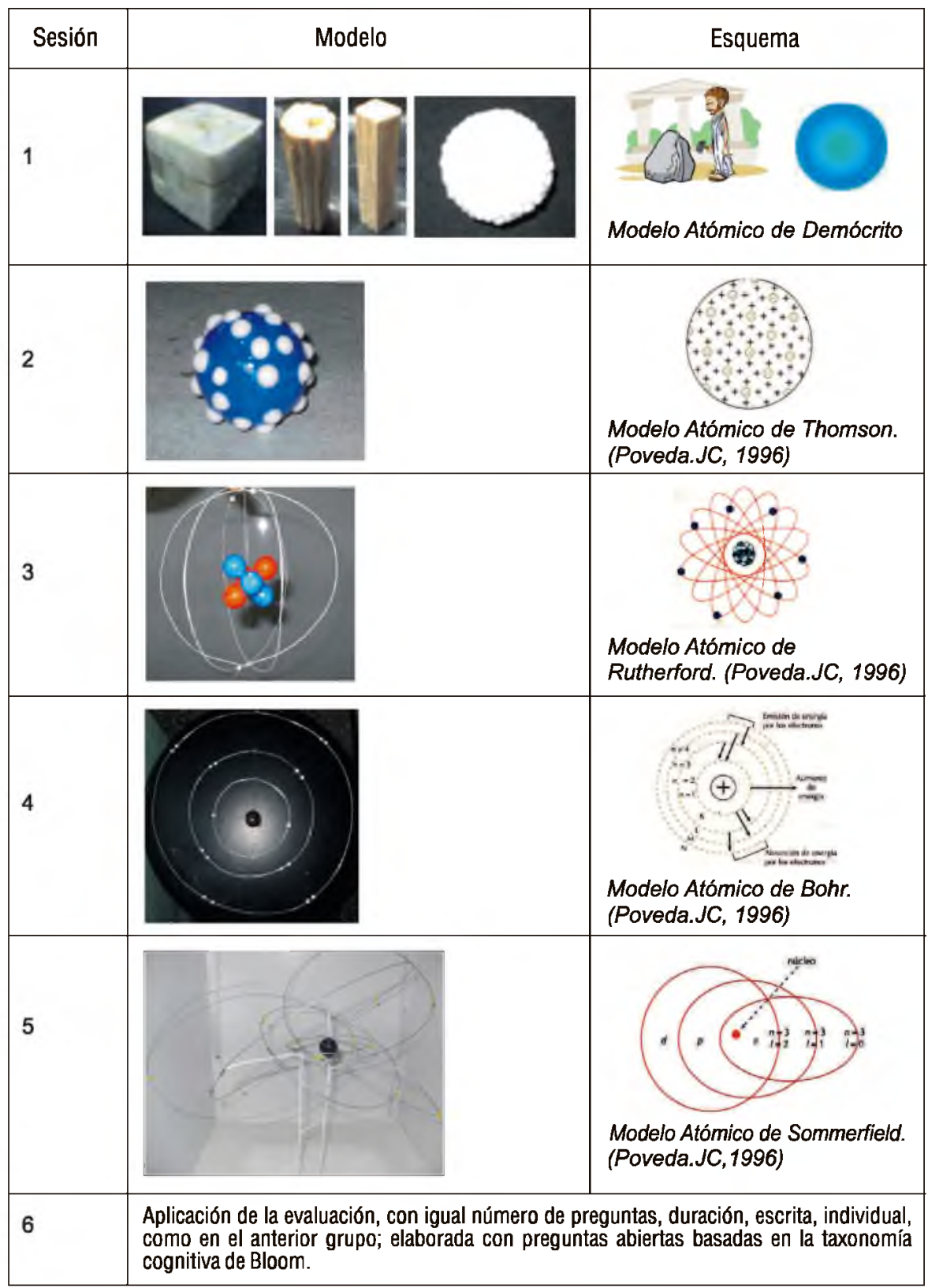


Se seleccionaron 17 estudiantes para el grupo GTIC, porque este era el número de computadores activos en la sala de informática de la institución y se dio prioridad al trabajo con herramientas y condiciones reales de una institución pública. El mismo número de estudiantes fue empleado en el grupo GFT con el objetivo de realizar una comparación directa entre grupos.

\section{Evaluación del Aprendizaje}

Para valorar el aprendizaje de los estudiantes en las diferentes estrategias, fueron sometidos a una prueba escrita constituida por 12 preguntas específicas del tema, las cuales se clasificaron de acuerdo con los niveles de pensamiento de la Taxonomía Cognitiva de Bloom, encontrando: preguntas de conocimiento, que determinan la capacidad para recordar información previamente aprendida (5 preguntas); preguntas de comprensión, que determinan la capacidad para entender y apropiarse lo aprendido (2 preguntas); preguntas de aplicación, que determinan la capacidad para transferir y utilizar datos y leyes para completar un problema o tarea ( 2 preguntas); preguntas de análisis, que determinan la capacidad para distinguir, clasificar y relacionar evidencias o estructuras de un hecho o de una pregunta, hacer preguntas, elaborar hipótesis ( 2 preguntas); y preguntas de síntesis, que determinan la capacidad para crear, integrar, combinar ideas, planear, y proponer nuevas maneras de hacer (1 pregunta).

Los valores dados para cada respuesta fueron: 1,0 para respuestas correctas y bien argumentadas; 0,5 para respuestas medianamente correctas pero sin argumentos o respuestas que demuestran conocimientos del tema pero no son correctas; $y, 0,0$ para respuestas incorrectas y donde no se demuestra conocimiento del tema.

La valoración fue realizada de forma neutral: todas las evaluaciones fueron reunidas, numeradas y evaluadas desconociendo el estudiante y el grupo experimental al que pertenecían.

\section{Análisis Estadístico}

En la última sesión, se recolectó la información y se consignó en un cuadro de resultados. Debido a que las variables son categóricas los resultados se consignaron en una tabla de contingencia donde se cruzaron las variables de interés que para este caso son las estrategias (GT, GFT y GTIC) y la calificación, dejando como réplicas del experimento la variable pregunta.

Se aplicó entonces, una prueba de chi-cuadrado para independencia, para probar si la calificación está asociada con la estrategia. Como hipótesis nula (H0), se consideran que los datos son independientes y como hipótesis alternativa (H1), que existe asociación entre los datos.
Si el valor de $p$ obtenido es $<0.05$, entonces se rechaza la hipótesis nula, es decir, existe asociación en los datos lo que permite deducir cual estrategia estimulo un mejor desempeño de los estudiantes.

Todos los análisis se llevaron a cabo usando el paquete estadístico R, de libre distribución (R-DevelopmentCore-Team, 2008).

\section{Consideraciones Éticas}

Una vez que el estudio fue aprobado y avalado por las directivas de la institución, se reunieron los estudiantes del grado décimo y se realizó la invitación a participar, la investigación fue realizada entonces con aquellas personas interesadas y que accedieron a firmar el consentimiento informado. Se les aviso igualmente que eran libres de abandonar el estudio en cualquier momento y que las notas obtenidas en las pruebas no tendrían valor sobre la nota de la disciplina.

Al final del estudio los estudiantes recibieron las clases correspondientes del tema abordado, con la metodología previamente establecida por el docente de la institución.

\section{Resultados}

Exceptuando la pregunta nueve, la aplicación de la prueba de chi - cuadrado en cada una de las 12 preguntas, permitió encontrar asociación entre la estrategia y la calificación obtenida por los estudiantes. Los resultados consolidados muestran que los estudiantes que usaron la estrategia de modelos físicos tridimensionales (GFT) presentaron el mayor porcentaje de respuestas correctas, el mayor porcentaje de medianamente correctas y el menor porcentaje de respuestas con valoración 0. El segundo grupo con el mejor desempeño fue el grupo TIC. Por otro lado los estudiantes pertenecientes al grupo con metodología tradicional presentaron las calificaciones más bajas. Además, también presento el mayor porcentaje de respuestas incorrectas y el menor porcentaje de respuestas correctas (Tabla 3). Esta tendencia es mostrada con el gráfico de análisis de correspondencia donde se observa que el grupo TIC y el grupo GFT están direccionados hacia las respuestas con valor de 1,0 (Figura, 1).

\section{Respuestas a las preguntas de Conocimiento}

Los estudiantes del grupo TIC presentaron un mejor desempeño en tres preguntas de conocimiento, mientras que los estudiantes que trabajaron con modelos físicos tridimensionales se destacaron en dos (resultados no mostrados). Las respuestas menos favorables fueron las dadas por el grupo tradicional. Consolidando estos resultados y llevándolos a porcentajes el mejor desempeño fue del grupo TIC y el grupo GFT (Figura 2) 
Tabla 3. Resultados consolidados de los valores asignados a las respuestas de una evaluación de 12 preguntas aplicada a tres grupos de estudiantes que aprendieron con igual número de estrategias pedagógicas.

GFT = Grupo de Modelos Físicos Tridimensionales,

GTIC = Grupo de Tecnologías de la y de la Comunicación,

GT = Grupo de docencia tradicional y análisis de chi quadrado.

\begin{tabular}{|l|l|l|l|}
\hline \multirow{2}{*}{ Estrategia } & \multicolumn{3}{|l|}{ Valoración } \\
\cline { 2 - 4 } & 0 & 0.5 & 1 \\
\hline GFT & $38,72 \%(n=79)$ & $48 \%(n=98)$ & $13,23 \%(n=27)$ \\
\hline GTIC & $43,13 \%(n=88)$ & $44,11 \%(n=90)$ & $12,74 \%(n=26)$ \\
\hline GT & $86,74 \%(n=386)$ & $10,56 \%(n=47)$ & $12,6 \%(n=12)$ \\
\hline
\end{tabular}

Pearson's Chi-squared test

Data: Estrategia de enseñanza

$\mathrm{X}$-squared $=196.8618, \mathrm{df}=4, \mathrm{p}-$ value $<2.2 \mathrm{e}-16$

Figura 1. Análisis de correspondencia de las respuestas de una evaluación de 12 preguntas de un grupo de estudiantes en el que se usaron tres diferentes estrategias pedagógicas.

GFT = Grupo de Modelos Físicos Tridimensionales,

GTIC = Grupo de Tecnologías de la y de la Comunicación,

GT = Grupo de docencia tradicional.

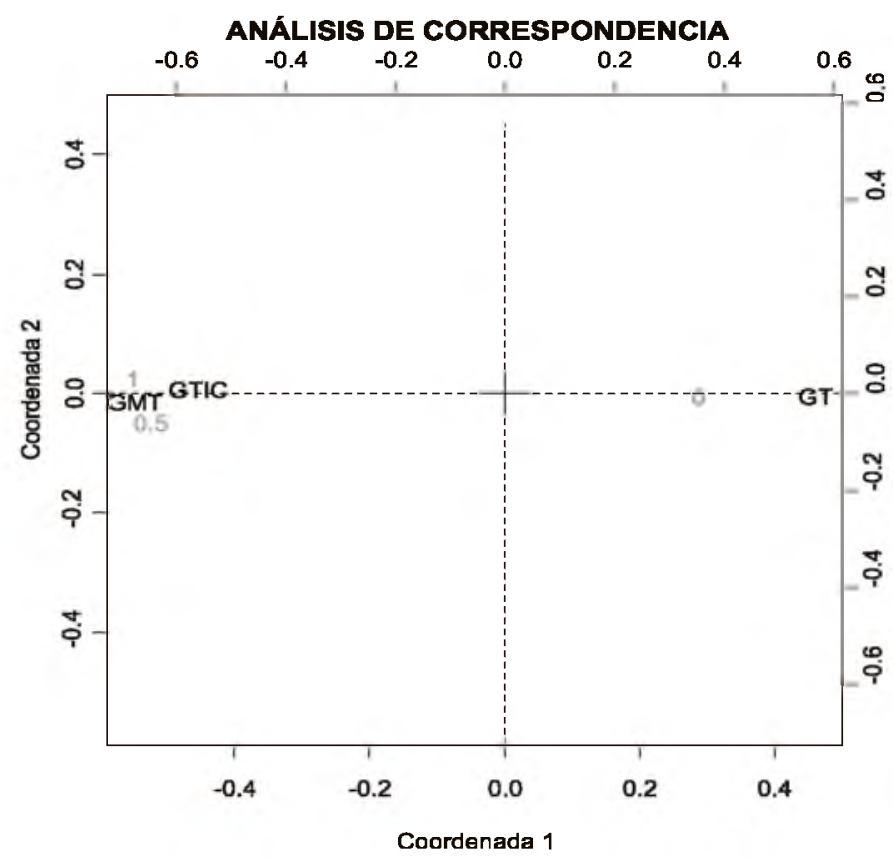

Figura 2. Valores en porcentaje de las respuestas incorrectas, parcialmente correctas y correctas a preguntas de tipo conocimiento aplicadas a estudiantes de secundaria. Posterior al uso de tres metodologías diferentes para la enseñanza del tema de estructura atómica.

GFT = Grupo Modelos Físicos Tridimensionales $(n=17)$,

GTIC $=$ Grupo Tecnología de la Información y de la Comunicación $(\mathrm{n}=17)$,

GT = Grupo de Docencia Tradicional $(\mathrm{n}=37)$.

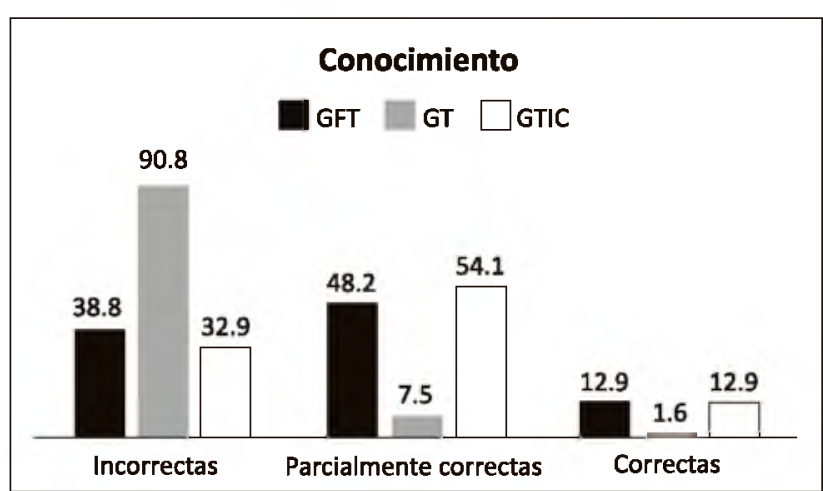

\section{Respuestas a las preguntas de Comprensión}

El mejor desempeño en esta respuesta fue de los estudiantes del GTIC, algunos de estos estudiantes consiguieron desarrollar adecuadamente las preguntas. Ya un alto porcentaje de estudiantes del GFT, consiguió responderla correctamente sin sustentar la respuesta o demostrar conocimientos sobre la pregunta sin tener la respuesta acertada. El grupo tradicional presento el mayor porcentaje de respuestas incorrectas (Figura 3).

Figura 3. Valores en porcentaje de las respuestas incorrectas, parcialmente correctas y correctas a preguntas de tipo comprensión aplicadas a estudiantes de secundaria. Posterior al uso de tres metodologías diferentes para la enseñanza del tema de estructura atómica.

GFT = Grupo Modelos Físicos Tridimensionales $(\mathrm{n}=17$ ),

GTIC $=$ Grupo Tecnología de la Información y de la Comunicación ( $\mathrm{n}=17$ ),

GT = Grupo de Docencia Tradicional ( $\mathrm{n}=37)$.

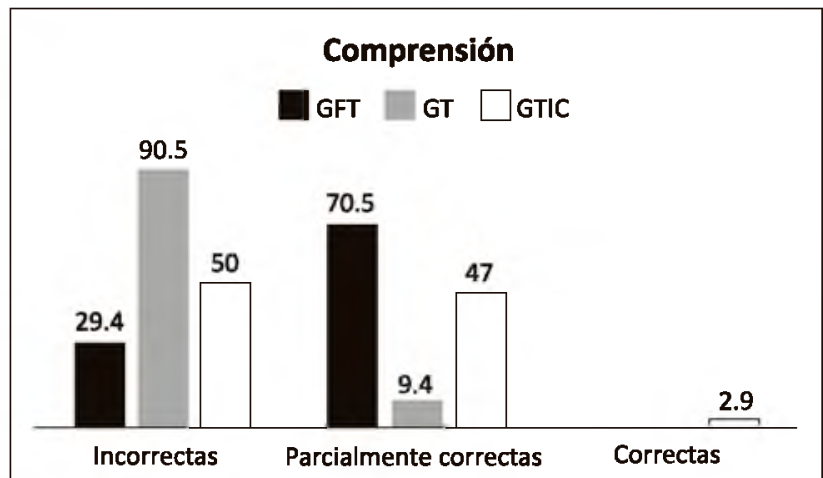




\section{Respuestas a las preguntas de aplicación}

Las 2 preguntas de aplicación fueron mejor respondidas por el grupo GFT, las respuestas de los estudiantes del grupo GTIC, le siguieron y con el desempeño más modesto se encontró al grupo GT (Figura 4).

Figura 4. Valores en porcentaje de las respuestas incorrectas, parcialmente correctas y correctas a preguntas de tipo aplicación aplicadas a estudiantes de secundaria. Posterior al uso de tres metodologías diferentes para la enseñanza del tema de estructura atómica.

GFT = Grupo Modelos Físicos Tridimensionales $(n=17)$, GTIC = Grupo Tecnología de la Información y de la Comunicación $(n=17)$,

GT = Grupo de Docencia Tradicional $(n=37)$.

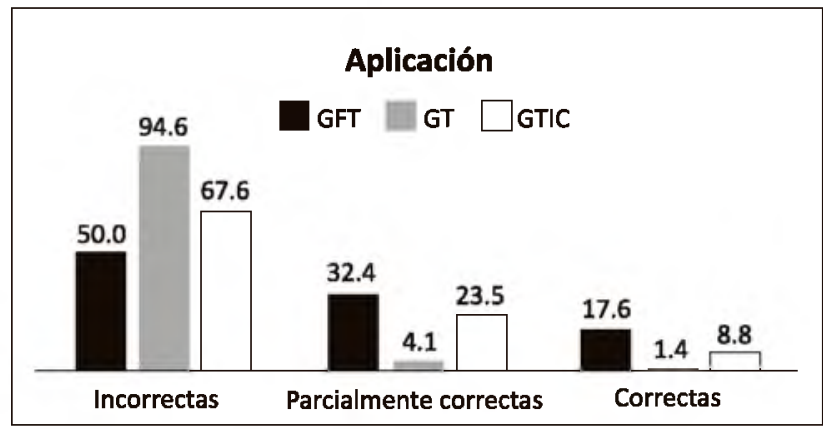

Respuestas a las preguntas de Análisis

En las dos preguntas de este nivel de conocimiento el grupo GTIC obtuvo los mejores resultados, seguido del grupo GFT. Estos dos grupos presentaron un porcentaje igual en las respuestas medianamente acertadas. El GT presento el mayor porcentaje de respuestas incorrectas (Figura 5).

Figura 5. Valores en porcentaje de las respuestas incorrectas, parcialmente correctas y correctas a preguntas de tipo análisis aplicadas a estudiantes de secundaria. Posterior al uso de tres metodologías diferentes para la enseñanza del tema de estructura atómica.

GFT = Grupo Modelos Físicos Tridimensionales $(\mathrm{n}=17)$, GTIC $=$ Grupo Tecnología de la Información y de la Comunicación $(\mathrm{n}=17)$,

GT = Grupo de Docencia Tradicional $(n=37)$.

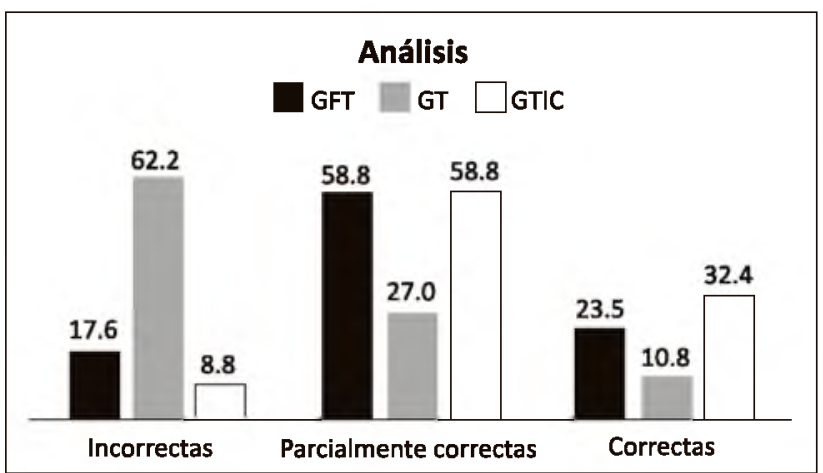

\section{Respuestas a las pregunta de síntesis}

En esta pregunta los tres grupos presentaron los resultados más modestos. Solamente el grupo GFT consiguió respuestas correctas. Es notable que el desempeño más modesto fuera el del grupo GTIC (Figura 6).

Figura 6. Valores en porcentaje de las respuestas incorrectas, parcialmente correctas y correctas a preguntas de tipo síntesis aplicadas a estudiantes de secundaria. Posterior al uso de tres metodologías diferentes para la enseñanza del tema de estructura atómica.

GFT = Grupo Modelos Físicos Tridimensionales ( $n=17)$,

GTIC = Grupo Tecnología de la Información y de la Comunicación $(\mathrm{n}=17)$,

GT = Grupo de Docencia Tradicional $(n=37)$.

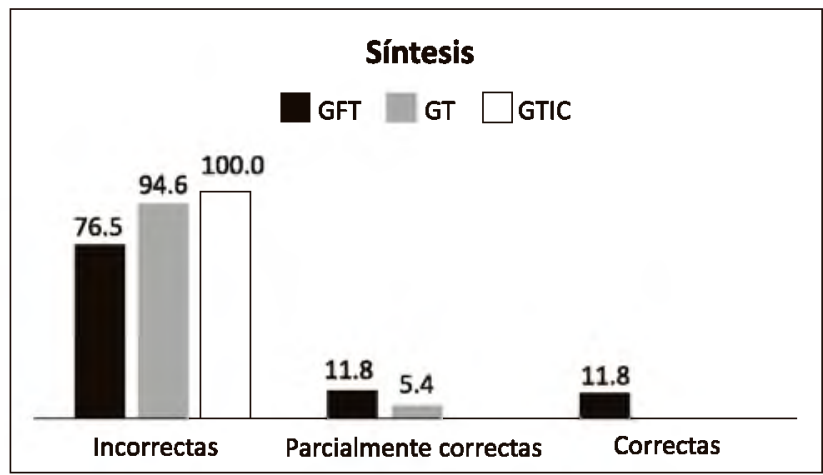

\section{Discusión}

La búsqueda de estrategias por trasmitir conceptos que son abstractos o de difícil comprensión es un reto para los docentes. En esta investigación es evidente que el uso de alternativas a la docencia tradicional es atractiva y realmente estimula el aprendizaje de los estudiantes lo que se refleja en los resultados.

Se ha reportado que los estudiantes de educación básica, consideran beneficioso el empleo de ayudas didácticas con alguna frecuencia (Duarte JE, 2009). Sin embargo, gran parte de los docentes enseña representaciones de moléculas en libros de texto, y asumen que todos los estudiantes pueden convertir una imagen de dos dimensiones en un modelo mental tridimensional. No obstante, se ha comprobado que existen diferencias en la capacidad de crear estructuras tridimensionales mentales entre individuos, principalmente entre hombres y mujeres (Harris, et al., 2009).

Está claro que el uso de material didáctico para introducir conceptos durante las clases promueve la creatividad de los estudiantes, ya que se tiene una mayor libertad de aprender, se coopera con los compañeros y se buscan soluciones de problemas planteados (Harris, et al., 2009) (Orlando et al., 2009) (Duarte JE, 2009). De esta forma, los estudiantes que han manipulado modelos didácticos presentan un entendimiento mejor y 
más completo de los conceptos abstractos, lo que se refleja en un mejor desempeño a la hora de resolver problemas de química (Wu \& Shah, 2004).

Otros estudiantes que tuvieron un desempeño aceptable, fueron los resultados que realizaron las sesiones trabajando con ayuda de la web 2.0. Estudios previos sugieren la utilidad de las herramientas virtuales como soporte al trabajo independiente que facilita la comprensión de temas específicos (Boude Figueredo OR, 2007) (Liu, 2007). De forma similar, otras investigaciones han comparado sistemas de docencia tradicional con alternativas basadas en la web 2.0, notando una mejoría en la comprensión de los estudiantes que mantienen sus clases de forma interactiva. Aunque estas nuevas metodologías exigen cambiar las tradicionales, es evidente una mejor comprensión de los temas cuando se modifica de un modelo centrado en el docente por uno donde el estudiante es participante activo (McDaniel, Lister, Hanna, \& Roy, 2007).

Resulta inquietante y quizá preocupante que el desempeño más modesto haya sido el de los estudiantes que participaron de la docencia tradicional (GT), modelo ampliamente manejado en las instituciones públicas. Se conoce que la mayoría de los estudiantes han recibido la información de manera pasiva en clases centradas en un docente que trasmite la información, y han organizado la información en formatos que conducen a la memorización pero no son apropiados para construir modelos conceptuales integrados (Modell, 1996).

Un aspecto que merece análisis es el número de estudiantes por aula, sin duda los grupos menos numerosos de estudiantes generalmente reciben un contacto más personalizado, sin embargo en los grupos GTIC y GFT, primo mucho el trabajo independiente y activo y no simplemente recibieron el contenido de forma pasiva, consideramos que más que el número de estudiantes, influyo notablemente el hecho de que las ayudas didácticas, para los grupos GTIC y GFT, permitieron sacar el concepto de lo abstracto y permitieron hacer el concepto palpable y tangible.

Otro punto importante es que a pesar de que se intenta homogenizar todas las variables, los estudiantes son bastante heterogéneos proceden de núcleos familiares con características propias lo que seguramente influye en el desempeño académico y en los resultados del estudio.

Se concluye finalmente que para mejorar el aprendizaje se deben introducir en las clases que abordan conceptos celulares y moleculares modelos tridimensionales, o se su defecto animaciones generadas por computador, que permitan una mejor percepción y comprensión por parte del estudiante, esto permite un ambiente activo, y autónomo lo que mejora su capacidad de respuesta ante problemas planteados. El estudio demostró que estas nuevas estrategias se pueden hacer en condiciones reales, los materiales empleados son de fácil obtención y de bajo costo, por otro lado el trabajo en computador se puede desarrollar en un equipo estándar con conexión convencional a internet.

Este nuevo enfoque es un reto ya que busca cambiar paradigmas tradicionales el sistema actual de educación promueve el papel pasivo de los estudiantes, el reto de direccionar ciertas disciplinas hacia un ambiente activo es incómodo y en ocasiones intimidante, pero es un cambio que se debe asumir.

\section{Agradecimientos}

Los autores expresan sus agradecimientos a los jóvenes, directivas de la institución y docentes que gentilmente concordaron en participar en esta investigación. Este trabajo es resultado de las investigaciones adelantadas por Benjamín Dussan Díaz como conclusión de la Maestría en Educación: Diseño, Gestión y Evaluación Curricular, de la Facultad de Educación de la Universidad Surcolombiana.

\section{Referencias Bibliográficas}

Adúriz-Bravo, Agustín, \& Izquierdo-Aymerich, Mercè. (2009). Un modelo de modelo científico para la enseñanza de las ciencias naturales. Revista electrónica de investigación en educación en ciencias, 40-49.

Boude Figueredo OR, Regalado LG. (2007). Nuevas tecnologías aplicadas a la educación: una experiencia en la enseñanza de la genética. Educación y Educadores, 10(2), 165-173.

Cardenas, F.A. (2006). Dificultades de Aprendizaje en Química: Caracterización y Búsqueda de Alternativas Para Superarlas. Ciência \& Educaçấo, 12(3), 333-346.

Duarte JE, Fernández-Morales FH, Angarita-Velandia MA. (2009). Relación del material didáctico con la enseñanza de ciencia y tecnología (Vol. 11).

Escalona, José, \& Fontal, Bernardo. (2008). El paradigma del pensamiento complejo en la didáctica de la química: Una visión desde el átomo a la materia. Educere, 12, 23-29.

Harris, M. A., Peck, R. F., Colton, S., Morris, J., Chaibub Neto, E., \& Kallio J. (2009). A combination of hand-held models and computer imaging programs helps students answer oral questions about molecular structure and function: a controlled investigation of student learning. CBE Life Sci Educ, 8(1), 29-43.

Kramer, I. M., Dahmani, H. R., Delouche, P., Bidabe, M., \& Schneeberger, P. (2012). Education catching up with science: preparing students for three-dimensional literacy in cell biology. CBE Life Sci Educ, 11(4), 437-447.

Liu, D. (2007). Seeing cells on the web. CBELife Sci Educ, 6(1), 21-24.

McDaniel, C. N., Lister, B. C., Hanna, M. H., \& Roy, H. (2007). Increased learning observed in redesigned introductory biology course that employed web-enhanced, interactive pedagogy. CBE Life Sci Educ, 6(3), 243-249.

Mercè, Izquierdo Aymerich. (2004). Un nuevo enfoque de la enseñanza de la química: contextualizar y modernizar. An. Asoc. Quím. Argent, 92(4-6), 115-136.

Modell, H. I. (1996). Preparing students to participate in an active learning environment. Am J Physiol, 270(6 Pt 3), S69-77.

O'Dowd, D. K., \& Aguilar-Roca, N. (2009). Garage demos: using physical models to illustrate dynamic aspects of microscopic biological processes. CBE Life Sci Educ, 8(2), 118-122

Orlando, Tereza Cristina, Lima, Adriene Ribeiro, Silva, Ariadne Mendes da, Fuzissaki, Carolina Nakau, Ramos, Cíntia Lacerda, Machado, Daisy, . Tréz, Thales de A. e. (2009). PLANEJAMENTO, MONTAGEM E APLICAÇĀO DE MODELOS DIDĀTICOS PARA ABORDAGEM DE BIOLOGIA CELULAR E MOLECULAR NO ENSINO MÉDIO POR GRADUANDOS DE CIENCIAS BIOLÓGICAS (Vol. 7).

Pérez Miranda, R. y Gallego-Badillo, R. (1995). Corrientes constructivistas. Cooperativa Editorial Magisterio.

R-Development-Core-Team. (2008). A language and environment for statistical computing. R Foundation for Statistical Computing.

Wu, Hsin-Kai, \& Shah, Priti. (2004). Exploring visuospatial thinking in chemistry learning. Science Education, 88(3), 465-492. doi: $10.1002 /$ sce. 10126 . 